\title{
Françoise Massardier-Kenney, Traduire Valvèdre
}

\section{Sonia Gerolimich}

\section{(2) OpenEdition}

\section{Journals}

\section{Édition électronique}

URL : http://journals.openedition.org/studifrancesi/27781

DOI : 10.4000/studifrancesi.27781

ISSN : 2427-5856

\section{Éditeur}

Rosenberg \& Sellier

\section{Édition imprimée}

Date de publication : 31 décembre 2006

Pagination : 621-622

ISSN : 0039-2944

\section{Référence électronique}

Sonia Gerolimich, «Françoise Massardier-Kenney, Traduire Valvèdre », Studi Francesi [En ligne], 150 (L III) | 2006, mis en ligne le 30 novembre 2015, consulté le 08 novembre 2020. URL : http:// journals.openedition.org/studifrancesi/27781; DOI : https://doi.org/10.4000/studifrancesi.27781

\section{Ce document a été généré automatiquement le 8 novembre 2020}

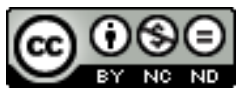

Studi Francesi è distribuita con Licenza Creative Commons Attribuzione - Non commerciale - Non opere derivate 4.0 Internazionale. 


\title{
Françoise Massardier-Kenney, Traduire Valvèdre
}

\author{
Sonia Gerolimich
}

\section{RÉFÉRENCE}

FRANÇOISE MASSARDIER-KENNEY, Traduire Valvèdre, George Sand Studies, vol. 23, 2004, pp. 62-69.

1 Plus qu'un commentaire sur la façon de traduire Valvèdre (1861), roman de George Sand, qui a été peu considéré, tant par la critique que par le public, alors qu'il avait connu un certain succès lors de sa parution, cet article, court mais dense, est avant tout une réflexion sur la façon dont le traducteur doit se comporter devant toute œuvre ainsi qu'une analyse de ce qui fait la spécificité du style de George Sand.

2 En effet Françoise Massardier-Kenney, qui fait un tour d'horizon des différentes traductions en anglais et regrette la quasi-absence de traductions de George Sand dignes de ce nom, considère que l'activité de traduire suppose que le traducteur ait étudié au préalable les caractéristiques stylistiques de l'auteur à traduire afin de les reproduire. Traduire équivaut à "restituer" un style, ce qui fait sa spécificité et non essayer d'“arranger" la langue d'un auteur comme c'est souvent le cas. On ne peut se permettre de couper les phrases, retrancher des mots, voire éliminer des passages. Il faut restituer toute œuvre avec fidélité pour qu'elle soit connue et "reconnue" pour ce qu'elle est.

D'ailleurs, Françoise Massardier-Kenney, qui a entrepris de traduire Valvèdre en anglais, en particulier parce qu'il s'agit «d'une œuvre capitale pour qui veut comprendre la modernité de George Sand», «son constructivisme», «ses innovations au niveau narratologique» (p. 64), s'attache à montrer certains aspects qui caractérisent le style de Sand dans ce roman et que le traducteur se doit de reproduire, comme la nécessité de recréer le "réseau signifiant" de cette œuvre; c'est le cas pour la série de métaphores sur la femme liées à la botanique, qui mettent l'accent sur “développement naturel" de 
la femme, ou pour le réseau métaphorique lié au champ juridique et financier qui a la fonction de "légitimer le divorce", autre thème crucial de Valvèdre. Il en est de même pour les démonstratifs qu'il convient de ne pas effacer en anglais comme c'est souvent la tendance puisque pour George Sand c'est une façon de "rapprocher le lecteur du monde du roman", ainsi que pour les abondantes expressions impersonnelles, comme "il me semble", indispensables pour souligner la difficulté que les hommes ont de percevoir le monde tel qu'il est réellement.

4 En outre, le traducteur, s'il veut rester fidèle au texte qu'il traduit, doit également consulter ce qu'on appelle les "textes parallèles", c'est-à-dire des œuvres d'auteurs écrivant dans la langue d'arrivée (en l'occurrence l'anglais dans le cas de l'auteur), contemporains de l'écrivain que l'on veut traduire et qui ont subi son influence. C'est le cas, parmi d'autres, de James, grand admirateur de George Sand qui utilise un style et des sujets proches de ceux de cette dernière. A titre d'exemple, l'auteur montre que le projet de "sortir de soi" évoqué par George Sand dans Valvèdre se retrouve dans l'œuvre de James et correspond à l'expression "getting out of oneself", employée par ce dernier, et dont le traducteur peut tirer profit.

5 Ainsi, Françoise Massardier-Kenney, mettant en pratique les importants préceptes énoncés au départ sur la traduction, met en évidence plusieurs caractéristiques du style George Sand dans Valvèdre que le traducteur se doit de respecter et de recréer, telles que sa fluidité, la symbolique des noms, la présence de tout un "réseau signifiant" de métaphores, l'emploi constant de "doublets", tant d'exemples qui ont le mérite de nous amener à "redécouvrir" ce qui constitue le "canon sandien". 